\title{
Typhoid Splenic Abscess: A Case Report and Literature Review
}

\author{
Fahmi Yousef Khan (iD) ${ }^{1, *}$ \\ ${ }^{1}$ Department of Medicine, Hamad General Hospital, Doha, Qatar \\ "Corresponding author: MD Consultant, Department of Medicine Hamad General Hospital, P.O. Box: 3050, Doha, Qatar. Tel: +974-55275989, Fax: +974-44321276, Email: \\ fakhanqal@gmail.com
}

Received 2018 December 06; Revised 2018 December 26; Accepted 2018 December 29.

\begin{abstract}
Introduction: Typhoid splenic abscess is a rare clinical entity that can be fatal if left untreated. Herein, we reported a case of typhoid splenic abscess who responded to ultrasound-guided aspiration and antibiotic therapy.

Case Presentation: A 25-year-old Nepalese man with no significant medical history presented to the emergency department with fever and the left upper abdominal pain lasting for seven days. One month before his presentation to our hospital, he returned from an eight-week holiday in Nepal. Splenic abscess was diagnosed by abdomen ultrasound and enhanced-computerized tomography. The patient refused surgical intervention and ultrasound-guided abscess aspiration was performed while piperacillin-tazobactam was initiated. Blood and aspirated pus microscopy revealed Gram-negative bacilli and the culture grew Salmonella typhi, which was sensitive to piperacillin-tazobactam, ampicillin, ciprofloxacin, and ceftriaxone. Piperacillin-tazobactam was continued for 4 weeks with successful recovery.

Conclusions: This case highlights the importance of considering typhoid splenic abscess in the differential of fever and abdominal pain in returning travelers.
\end{abstract}

Keywords: Typhoid, Splenic Abscess, Splenectomy, Aspiration

\section{Introduction}

Splenic abscess is an unusual and potentially lifethreatening disease with a reported incidence of $0.14 \%$ $0.7 \%$ in one autopsy series and bacterial abscess is the most common (1). Splenic abscess due to Salmonella typhi (S. typhi) is unusual, most of them associated with liver abscess whereas isolated splenic abscess is very rare. In this report, we presented a case of isolated splenic abscess due to $S$. typhi who was treated by abscess aspiration and antibiotic therapy.

This case highlights the importance of considering typhoid splenic abscess in the differential diagnosis of fever and abdominal pain in returning travelers.

\section{Case Presentation}

A 25-year-old healthy Nepalese patient presented to the emergency department with fever and the upper left abdominal pain lasting for seven days. One month prior to his presentation to our hospital, he returned from an eight-week holiday in Nepal. On examination, the patient looked sick, feverish, with a temperature of $39^{\circ} \mathrm{C}$, a heart rate of 96 beats per minute and a respiratory rate of 18 breaths per minute. His blood pressure was 110/60 mmHg and the oxygen saturation was $96 \%$ in ambient air. Examination of the abdomen showed a palpable, tender spleen, while the remainder of his examination was unremarkable.

Laboratory tests revealed leucopenia $\left(3.4 \times 10^{9} / \mathrm{L}\right)$ and thrombocytopenia $\left(109 \times 10^{9} / \mathrm{L}\right)$. Blood chemistry, liver profile, and coagulation tests were within normal limits. Malaria parasite smear, urine analysis, and Brucella serologic investigation were negative. Hepatitis A IgM antibody, hepatitis $C$ antibody, hepatitis B markers, and antibodies to human immunodeficiency virus (HIV) were likewise negative. Blood samples were sent to the laboratory for Gram staining, culture, and susceptibility tests. Imaging study, including chest and abdominal X-ray were unremarkable, whereas abdominal ultrasonography showed enlarged spleen with multiple well-defined cystic focal lesions. Contrast-enhanced computerized axial tomography (CAT) of the abdomen revealed multiple abscesses in the spleen. The largest abscess measured approximately $1.8 \times$ $0.8 \mathrm{~cm}$ (Figure 1). The surgeon was consulted and recommended a splenectomy, but the patient refused this operation. Therefore, ultrasound-guided aspiration of the largest abscess was performed and a broad spectrum intra- 
venous antibiotic (piperacillin-tazobactam) was initiated empirically at a dose of $4.5 \mathrm{~g}$ every eight hours. Blood and aspirated pus microscopy revealed Gram negative bacilli meanwhile the culture grew Salmonella typhi, which was sensitive to ampicillin, ciprofloxacin, ceftriaxone, and piperacillin-tazobactam. Sickling test was performed and it was negative. On the 14th day of the hospitalization, the patient became afebrile while on piperacillin-tazobactam, his appetite improved, and showed a good general improvement. Repeating abdominal ultrasound revealed decline in the abscess sizes. Piperacillin-tazobactam was continued for 2 more weeks until full recovery.

\section{Discussion}

Typhoid fever remains an important public health problem in developing countries, as it is linked principally to poor hygiene such as inadequate sewage disposal and flooding as well as lack of clean drinking water (2). In Qatar, which is non-endemic, typhoid fever is common due to increasing travel to and immigration from typhoid-endemic countries.

Typhoid splenic abscess is a rare clinical entity, which was formerly believed to occur in immunocompromised patients $(3,4)$; however, our patient was immunocompetent, indicating the occurrence of this condition regardless of the patient's immune status.

Splenic abscess is one of the abdominal complications of the late treatment of typhoid fever, which is usually as a result of delayed diagnosis (4-6). In accordance with the current report, previous studies have demonstrated that (3-13) the clinical picture of splenic abscess is nonspecific and non-distinguishable from other febrile illness, which

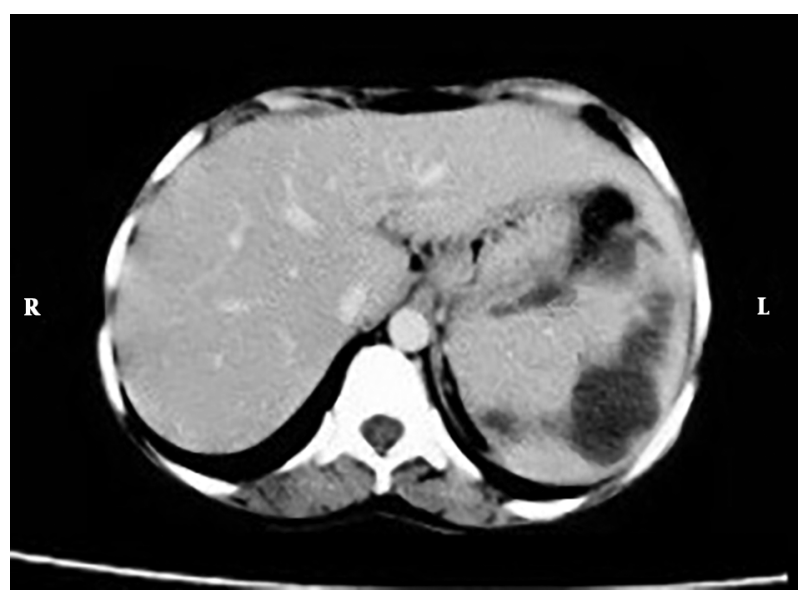

$\overline{\text { Figure 1. Abdomen CT showed multiple abscesses in the spleen. The largest abscess }}$ was measured approximately $1.8 \times 0.8 \mathrm{~cm}$. may leads to delayed diagnosis. Therefore, a high degree of suspicious is required for early and accurate detection of S. Typhi, to prevent its further invasion of different organs such as the spleen. In non-endemic areas such as Qatar, travel history is very helpful in formulating a suitable list of differential diagnosis in the early stage of this disease.

Abdomen ultrasonography and CT are very helpful in the early detection of the splenic lesion with abdomen $\mathrm{CT}$ being most sensitive and specific modality, moreover, microbiological study of blood and aspirated pus is essential to identify the etiological agent (3-5, 7-13). In our patient, these two modalities, as well as pus and blood cultures, played essential role in establishing the diagnosis. This study supports evidence from previous observations that typhoid splenic abscesses could present solitary/unilocular lesions $(3,12,13)$ or multiple/multilocular lesions $(4,5,7,8,10,11)$.

It is postulated that splenic abscess occurrence requires preexisting splenic tissue injury and bacteremia. However, typhoid splenic abscess can be formed by different mechanisms, including overwhelming bacteremia in immunocompromised individuals, patients whose spleen architectures have already been altered (e.g. splenic infarction in sickle cell disease or vasculitis), and an extension of infection from contiguous areas (e.g. intestinal perforation) (13). Our patient was immunocompetent and most probably he developed the abscess as a result of overwhelming bacteremia.

Treatment of typhoid splenic abscess is controversial because no randomized trials were conducted. Splenectomy along with antibiotics is regarded as the treatment of choice by many physicians $(5,7,8,11,13)$; however, our case, as well as other cases, showed that percutaneous abscess aspiration with appropriate antibiotics coverage could be as effective as splenectomy $(3,4,10,12)$. Furthermore, there is no consensus on the duration of antibiotic therapy, which should be adapted to each case. Our patient showed an excellent response to a 28-day antibiotic therapy.

In conclusion, typhoid splenic abscess is a rare clinical entity with nonspecific clinical picture. Early diagnosis is valuable to decrease the morbidity and mortality. CT scan and microbiological study of blood and aspirated components are useful diagnostic modalities to establish the diagnosis. No study is available to determine the most effective treatment of typhoid splenic abscess or the duration of antibiotics and we advise the physicians to individualize the management carefully.

\section{Footnotes}

Conflict of Interests: There are no conflicts of interest. 
Funding/Support: None declared.

Patient Consent: The authors certify that they have obtained all appropriate patient consent forms to publish this article.

\section{References}

1. Ferraioli G, Brunetti E, Gulizia R, Mariani G, Marone P, Filice C. Management of splenic abscess: Report on 16 cases from a single center. Int J Infect Dis. 2009;13(4):524-30. doi: 10.1016/j.ijid.2008.08.024. [PubMed: 19070526].

2. Ochiai RL, Acosta CJ, Danovaro-Holliday MC, Baiqing D, Bhattacharya SK, Agtini MD, et al. A study of typhoid fever in five Asian countries: Disease burden and implications for controls. Bull World Health Organ. 2008;86(4):260-8. doi: 10.2471/BLT.06.039818. [PubMed: 18438514]. [PubMed Central: PMC2647431].

3. Shamanna P, Ravindran J, Sethumadhavan M. Salmonella typhi causing splenic abscess - a rare case. Nat J Lab Med. 2017;6:MC01-3. doi: 10.7860/njlm/2017/22754:2187.

4. Bhongle NN, Nagdeo NV, Thombare VR. A splenic abscess which was caused by Salmonella typhi in a non sickler patient: A rare case finding. J Clin Diagn Res. 2013;7(3):537-8. doi: 10.7860/JCDR/2013/4563.2816. [PubMed: 23634415]. [PubMed Central: PMC3616575].

5. Piplani S, Ramakrishna Dr, Nandi B, Ganjoo RK, Madan R, Chander BN. Two cases of Salmonella splenic abscess. Med J Armed Forces India. 2006;62(1):77-8. doi: 10.1016/S0377-1237(06)80167-X. [PubMed: 27407854]. [PubMed Central: PMC4923292].
6. Miller SL, Hohmann EL, Pegues DA. Salmonella (including Salmonella typhi). In: GL Mandell GL, Bennett JE, Dolin R, editors. Principles and practice of infectious diseases. 4th ed. NY: Churchill Livingstone; 1995. p. 2013-32.

7. Bhatia M, Lohar H, Nirhale DS, Tomar V. Splenic abscess: a rare presentation. Egypt J Intern Med. 2015;27:78-9. doi: 10.4103/1110-7782.159477.

8. Kalyanwat AS, Jain S. Enteric fever presenting as splenic abscess: A rare presentation of enteric fever. OA Case Rep.1993;3(7):68.

9. Lee WS, Choi ST, Kim KK. Splenic abscess: A single institution study and review of the literature. Yonsei Med J. 2011;52(2):288-92. doi: 10.3349/ymj.2011.52.2.288. [PubMed: 21319348]. [PubMed Central: PMC3051211].

10. Handa A, Rajnikanth T, Bhartiya M, Sharma PK, Negi RS. Typhoid splenic abscess: A rarity in the present era. Sri Lankan J Infect Dis. 2015;5(2):96. doi: 10.4038/sljid.v5i2.8089.

11. Sharavanan P, Shanmugam D, Palraj KK, Antony T, Thayanidhi P. Salmonella typhi splenic abscess following blunt abdominal injury: A case report. J Clin Diagn Res. 2016;10(7):DD01-2. doi: 10.7860/JCDR/2016/19789.8156. [PubMed: 27630844]. [PubMed Central: PMC5020189].

12. Jaussaud R, Brasme L, Vernet-Garnier V, Deville JF. Splenic abscess complicating Salmonella typhi infection. Eur J Clin Microbiol Infect Dis. 2000;19(5):399-400. doi: 10.1007/s100960050504. [PubMed: 10898147].

13. Duggal S, Mahajan RK, Biswas NK, Chandel DS, Duggal N, Hans C. Splenic abscess due to Salmonella enterica Serotype typhi in a young adult. J Commun Dis. 2008;40(3):219-22. [PubMed: 19245162]. 